\title{
TRANSCRANIAL DIRECT CURRENT ELECTRICAL STIMULATION IMPROVES THE QUALITY OF LIFE ASSOCIATED TO VASCULAR CLAUDICATION OF A PATIENT WITH TAKAYASU ARTERITIS
}

Alexandre Moura dos Santos ${ }^{1, *}$, Rafael Giovanni Missé ${ }^{1}$, Lucas de Macedo dos Santos ${ }^{1}$, Abrahão Fonte Baptista², Clarice Tanaka $^{1}$, Rosa Maria R Pereira ${ }^{1}$, Julia Maria D’Andrea Greve ${ }^{1}$, Samuel Katsuyuki Shinjo ${ }^{1}$

1. Universidade de São Paulo, São Paulo (SP), Brazil. 2. Universidade Federal do ABC, São Paulo (SP), Brazil.

*Corresponding author: alexandrestos@usp.br

\section{BACKGROUND}

Recent studies have shown that vascular claudication can also be associated with increased central sensibilization, leading to impaired-quality of life and muscle function in patients with Takayasu arteritis (TAK). Several studies have shown that transcranial direct current electrical stimulation (tDCS) is able to modulate central perception. Due to the limited scope of existing research, we assessed the safety and impact of tDCS sessions on a TAK patient with vascular claudication and impaired quality of life and muscle function.

\section{CASE REPORT}

A white 47-year-old female patient with TAK diagnosis at the age of 20 (left subclavian artery occlusion, moderate stenosis in the left renal artery, left internal iliac artery and right external iliac artery, and abdominal aneurysm). Although she has complained of claudication in her right arm and the necessity to stop walking every 50 meters on a flat ground, she has never taken any drugs (glucocorticoids or immunosuppressive).
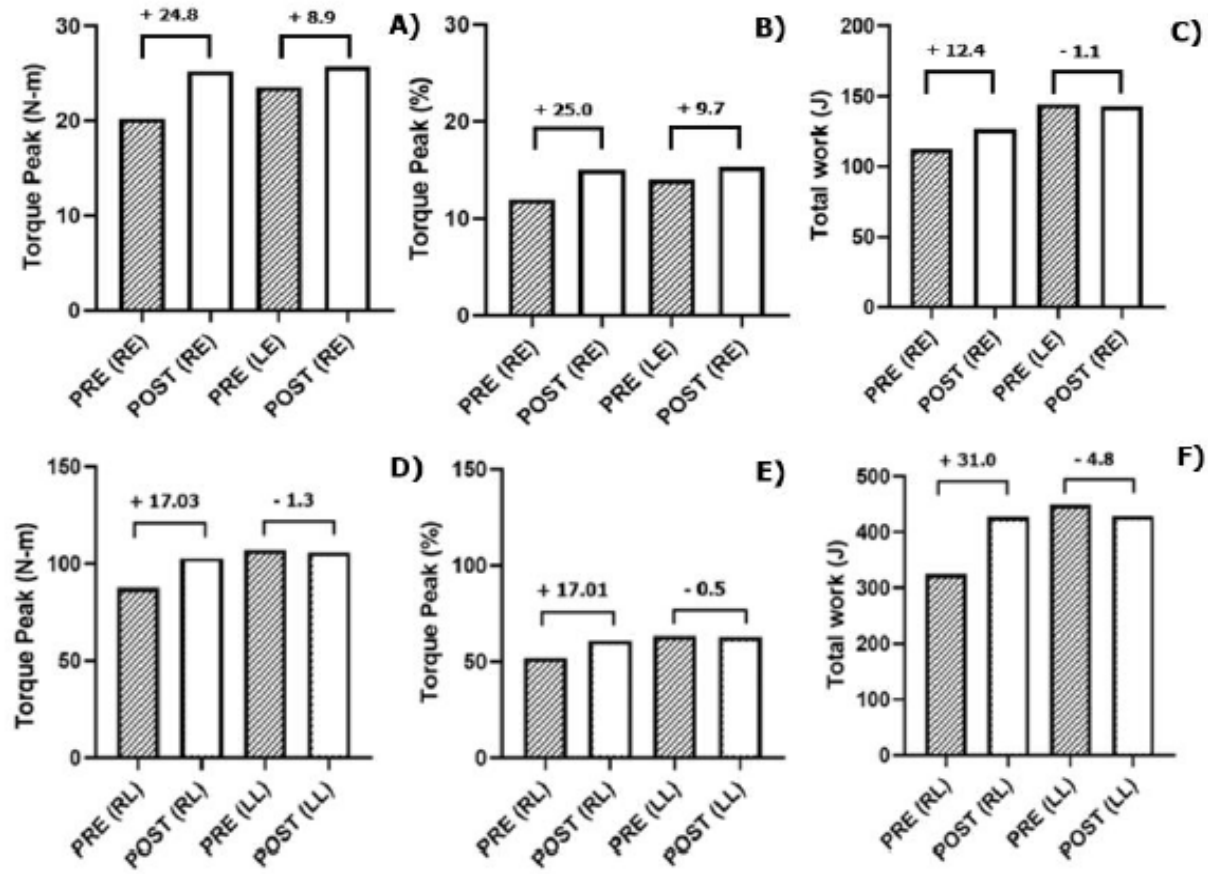

Figure 1. Strength parameters of right and left elbow (A-C) and right and left leg (D-F) 
After signing the informed consent, she underwent only three consecutive sessions of tDCS (with duration of 20 minutes for each session). A positively charged electrode was positioned at $\mathrm{C} 3$, and the negatively charged electrode was positioned in the supraorbital region FP2. Concomitantly, she underwent exercises characterized by 5 minutes on a treadmill, subsequently, bench press, squat, cable seated row, and leg press. At the end of the session, the patient was encouraged to walk 5 minutes on treadmill with low intensity (Borg scale), and the rest interval between exercise was 60 seconds. Strength was assessed using the isokinetic test in the baseline and one month after the end of the study.

After tDCS, the patient reported a significant improvement in her quality of life, her capacity to walk more than 300 meters on a flat ground without stopping, and in the functionality and strength of her right arm. After a month of protocol, the isokinetic test demonstrated an increase in her limbs peak torque without vascular claudication (Figure 1). During the following research neither side effects nor disease relapses were reported.

\section{CONCLUSION}

The tDCS appears to be safe and effective in reducing perceived vascular claudication, and in improving walking capacity and quality of life. Future studies are needed to corroborate this data.

\section{FUNDING}

Fundação de Amparo à Pesquisa do Estado de São Paulo (FAPESP Grant No. 2018/08735-3 to AMS; No. 2019/12155-5 to RGM; No. 2019/11776-6 to SKS). Conselho Nacional de Desenvolvimento Científico e Tecnológico (CNPq Grant No. 303379/2018-9 to SKS). 